\title{
System Identification Methodology Based on Absolute Acceleration Measurements
}

\author{
Yoshihiro Nitta and Akira Nishitani \\ Department of Architecture, Waseda University, 3-4-1, Okubo, Shinjuku, Tokyo, 169-8555, Japan \\ Tel/Fax:(+81)-5286-3286, E-mail:ynitta@aoni.waseda.jp
}

\begin{abstract}
This paper presents a relatively simple but quite effective identification methodology based on absolute acceleration response measurements. The proposed methodology consists of two stages. In the first stage, the first few modal vectors of a structure are estimated by utilizing the proper orthogonal decomposition technique. In the second stage, the bandpassfiltered input ground acceleration is initially estimated from the obtained modal vectors. Then, with the obtained input ground acceleration employed as an input, absolute acceleration responses subjected to the bandpass filter are used as an output vector in the ARX model-based identification. This ARX model provides more precise modal characteristics of a structure and updates the input ground acceleration time history. The concept of the proposed approach is presented and its validity is demonstrated by conducting numerical simulations and scale model experiments.
\end{abstract}

\section{Introduction}

During the last two decades or so system identification technique has been remarkably integrated into civil engineering field. In these days in particular, along with the recent development of structural control and health monitoring, system identification is expected to play more significant role at the stage of civil engineering.

One of the major approaches in system identification is based on certain mathematical model representing inputoutput relations. In this approach, both of the input and output data are measured except for the case in which an input is assumed to be a random white noise process. However, in a huge building structure, it is not a trivial task to take a synchronous measurement of the input and output. In addition, an accurate measurement of input such as an earthquake induced ground motion is likely to involve some difficulty because such input data sometimes involve other factors than those related to the pure input excitation source. Considering the above-mentioned difficulty with respect to the measurement of input data in a large building structure, it would be desirable if only several kinds of output data are measured and then the input is obtained from these measured output data. This paper presents a system identification methodology for a seismically excited building structure based on only the measurement of output data without involving any assumption with respect to input or any measurement of input data. The presented methodology consists of two stages. In the first stage, the modal vectors of a structure are estimated based on the limited number of measured output data. In the second stage, the information about input ground acceleration data is obtained from the modal vectors estimated in the first stage and then the ARX model-based identification is conducted by using the output data and the obtained information of ground acceleration. Finally, the methodology provides more precise information for the modal characteristics of a structure and updates the input ground acceleration time history. The validity of this methodology is demonstrated by conducting numerical simulations and scale model experiments.

\section{Identification of Modal Characteristics and Input Acceleration}

The first stage of the proposed methodology is to estimate the modal characteristics of a structure with the aid of the proper orthogonal decomposition (POD) $)^{1,2)}$. The POD technique is effectively utilized to detect the modal vectors based upon the measured absolute acceleration data for selected $M$ stories of a structure. However, these identified modal vectors from the absolute acceleration response data are likely to involve the effect of the ground accelerations. For the purpose of involving less effect of the ground acceleration, this paper deals with those absolute acceleration response data which are made to pass through the bandpass filters covering the range of each of the first few modal frequencies of a structure. This is because the responses in the vicinities of the modal frequencies are mainly due to the structural responses.

The correlation matrix of absolute acceleration response vector subjected to the bandpass filter corresponding to the $j$ thmode is given by

$$
\boldsymbol{R}_{j}=E\left[\boldsymbol{Y}_{j} \boldsymbol{Y}_{j}^{T}\right]
$$

with

$$
Y_{j}=\left[\begin{array}{lll}
y_{j}(0) & \cdots & y_{j}(N)
\end{array}\right]
$$

in which $y_{j}(k)$ represents the vector consisting of the $j$ th mode-corresponding-filtered absolute acceleration response at discrete time $k \Delta t$. Following the POD technique, the eigen vector corresponding to the largest eigen value of the correlation matrix $\boldsymbol{R}_{j}$ gives the $j$ th modal vector. The main purpose of the first step is to estimate the modal vectors for the selected modes.

Then, the methodology proceeds to the second step. 
Denoting $\phi_{i j}$ as the $i$ th component of the jth modal vector, the $j$ th modal absolute acceleration response of the $i$ th story can be written as:

$$
y_{i j}(k)=\phi_{i j} \zeta_{j}(k)+u_{j}(k)
$$

in which $y_{i j}(k)$ : component representing the $i$ th story response subjected to the bandpass filter with respect to the $j$ th modal frequency, $\zeta_{j}(k):$ acceleration response of the $j$ th modal coordinate and $u_{j}(k)$ : input ground acceleration subjected to the bandpass filter with respect to the jth modal frequency. Having the $j$ th modal absolute acceleration responses of two stories, say $m$ th and $n$th stories, the filtered input accelerations for the $j$ th modal frequency, $u_{j}(k)$, can be provided by

$$
u_{j}(k)=y_{m j}(k)-\phi_{m j} \times \frac{y_{m j}(k)-y_{n j}(k)}{\phi_{m j}-\phi_{n j}}
$$

In conducting the above calculation to estimate $u_{j}(k)$, the absolute acceleration data subjected to the bandpass filters employed for the second step are utilized.

In the second step, the ARX model-based identification technique ${ }^{3)}$ is employed by utilizing the estimated modal ground acceleration obtained from Eq.(3) for the first few modes. Then, the ARX model includes, $u_{j}(k)$, as an input and involves the filtered absolute acceleration vector, $\boldsymbol{y}_{\boldsymbol{j}_{j}}(k)$, as an output. Following the usual manner in architectural engineering field, in this paper, the ARX model is constructed so as to have the same coefficients for the autoregressive part with respect to any story and hence has the same modal frequencies and dampings, i.e. ${ }^{4)}$

$\boldsymbol{y}_{j}(k)=-\sum_{\ell=1}^{L} a_{\ell} \boldsymbol{y}_{j}(k-\ell)+\sum_{\ell=0}^{L} \boldsymbol{b}_{\ell} u_{j}(k-\ell)+\boldsymbol{e}(k)$

Based on the constructed ARX model formulation, Eq.(4), the pulse transfer function of the $i$ th story, $H_{i j}(z)$, is provided by

$$
H_{i j}(z)=\frac{b_{i 0}+b_{i 1} z^{-1}+\cdots+b_{i L} z^{-L}}{1+a_{1} z^{-1}+\cdots+a_{L} z^{-L}}=b_{i 0}+\sum_{\ell=1}^{L} \frac{r_{i \ell}}{1-P_{\ell} z^{-1}}
$$

where $b_{i \ell}$ : ith component of the coefficient vector $\boldsymbol{b}_{\ell}$.

From Eq.(5), the fundamental modal characteristics (modal frequency $f_{j}$, modal damping ratio $h_{j}$, and modal vector $\phi_{i j}$ ) are given, respectively, by Eqs.(6) - (8).

$$
\begin{aligned}
& f_{j}=\frac{\left|\log P_{j}\right|}{2 \pi \Delta t} \\
& h_{j}=\frac{-\log \left|P_{j}\right|}{2 \pi f_{j} \Delta t}
\end{aligned}
$$

$$
\phi_{i j}=\operatorname{Re}\left[\frac{2 r_{i j} \sqrt{1-h_{j}{ }^{2}}}{2 \pi f_{j} \Delta t\left(h_{j}-\sqrt{-1} \operatorname{sgn}\left[\operatorname{Im}\left[P_{j}\right]\right]\left(1-2 h_{j}{ }^{2}\right)\right.}\right]
$$

where $\Delta t$ : sampling time.

Reflecting the fact that the acceleration responses are measured only in $M$ stories, the participation vectors considering up to the first only $M$ modes are determined so as to satisfy the following conditions?

$$
\sum_{j=1}^{M} \beta_{j} \phi_{i j}=1.0
$$

Based on the identified modal frequencies, modal damping ratios and the estimated participation coefficients considering up to $M$ modes, the transfer function vector from the scalar input ground acceleration to the output absolute acceleration vector representing the $M$ stories responses can be calculated. By taking the Laplace transform of the measured acceleration data for the $n$th story, $x_{n}(s)$, and using the corresponding scalar element of the transfer function vector, $H_{n}(s)$, the Laplace transform of the input ground acceleration, $u(s)$, can be estimated in the following:

$$
u(s)=H_{n}(s)^{-1} x_{n}(s)
$$

where

$$
H_{n}(s)=1+\sum_{j=1}^{M} \frac{-s^{2}}{s^{2}+2 h_{j} \omega_{j} s+\omega_{j}^{2}} \beta_{j} \phi_{n j}
$$

Taking the inverse Laplace transform of the obtained $u(s)$ leads to the time history of input ground acceleration. Since in the proposed methodology the number of measured output affects the number of the estimated modes and hence influences the frequency range of the estimated transfer function; this means that the obtained input ground acceleration is the one subjected to the corresponding bandpass filter to the frequency range of thus identified transfer function.

\section{Numerical Results}

To demonstrate the effectiveness of the proposed approach, numerical simulations are conducted for a 20 -story building model. The first three fundamental frequencies of the employed 20-story building model are: $0.56 \mathrm{~Hz}, 1.38 \mathrm{~Hz}$ and $2.20 \mathrm{~Hz}$. The structural damping matrix is assumed to be proportional to the stiffness matrix so as to have the damping ratio of 0.02 with respect to the first mode. The absolute accelerations of 20th, 14th and 8th stories corresponding to the NS component of the 1940 El Centro earthquake with PGA = $2.0 \mathrm{~m} / \mathrm{sec}^{2}$ are assumed to be measured with the sampling time of $0.1 \mathrm{sec}$.

In conducting the proposed identification, the bandpass 
filters with the following passbands are employed in the first step utilizing POD technique: $0.3 \mathrm{~Hz} \sim 0.8 \mathrm{~Hz}$ for the first mode, $1.1 \mathrm{~Hz} \sim 1.6 \mathrm{~Hz}$ for the second mode and $2.0 \mathrm{H} \sim 2.4$ $\mathrm{Hz}$ for the third mode. These filters are assumed to be derived from certain pre-identification roughly estimating the natural frequencies. The purpose of the first step is to estimate the eigen vectors for the first three modes. Table 1 shows the modal vectors detected by POD. Since the output data are measured only for the 8th, 14th and 20th stories of this building, the identified modal vectors consist of the three components. The first, second and third components of each modal vector shown in Tables 1 and 3 correspond to the 8th, 14th and 20th stories, respectively.

By utilizing the information with respect to the modal vectors estimated in the first step, the input ground acceleration corresponding to each of the first three modes is evaluated based on the absolute acceleration data subjected to the bandpass filters with the following passbands for the second step: $0 \mathrm{~Hz} \sim 1.1 \mathrm{~Hz}$ for the first mode, $0.8 \mathrm{~Hz} \sim 1.9 \mathrm{~Hz}$ for the second mode and $1.6 \mathrm{~Hz} \sim 2.7 \mathrm{~Hz}$ for the third mode. Based on Eq.(3) the modal input ground accelerations are provided, which correspond to those subjected to the above filters. The ARX model is constructed based on the filtered absolute acceleration output with respect to the selected three stories

Table 1 Identified modal vectors

\begin{tabular}{cccc}
\hline Component & 1st mode & 2nd mode & 3rd mode \\
\hline 1st & 0.344 & 0.559 & 0.634 \\
& $\left(1.024^{*}\right)$ & $\left(0.962^{*}\right)$ & $\left(0.991^{*}\right)$ \\
2nd & 0.666 & 0.421 & -0.687 \\
& $\left(1.006^{*}\right)$ & $\left(1.005^{*}\right)$ & $\left(1.099^{*}\right)$ \\
3rd & 1.000 & -1.000 & 1.000 \\
& $\left(1.000^{*}\right)$ & $\left(1.000^{*}\right)$ & $\left(1.000^{*}\right)$ \\
\hline \multicolumn{4}{c}{ * the ratio of identified value to true value }
\end{tabular}

Table 2 Identified frequencies and dampings

\begin{tabular}{ccc}
\hline Mode & $\begin{array}{c}\text { Natural frequency } \\
{[\mathrm{Hz}]}\end{array}$ & Damping ratio \\
\hline 1 & 0.56 & 0.020 \\
& $\left(0.995^{*}\right)$ & $\left(1.000^{*}\right)$ \\
2 & 1.35 & 0.048 \\
& $\left(0.979^{*}\right)$ & $\left(0.980^{*}\right)$ \\
3 & 2.16 & 0.076 \\
& $\left(0.978^{*}\right)$ & $\left(0.974^{*}\right)$ \\
\hline \multirow{3}{*}{} & $*$ the ratio of identified value to true value
\end{tabular}

Table 3 Identified participation vectors

\begin{tabular}{cccc}
\hline Component & 1st mode & 2nd mode & 3rd mode \\
\hline 1st & 0.475 & 0.373 & 0.153 \\
& $\left(0.975^{*}\right)$ & $\left(0.913^{*}\right)$ & $\left(0.608^{*}\right)$ \\
2nd & 0.926 & 0.242 & -0.168 \\
& $\left(0.964^{*}\right)$ & $\left(0.826^{*}\right)$ & $\left(0.688^{*}\right)$ \\
3rd & 1.385 & -0.642 & 0.257 \\
& $\left(0.993^{*}\right)$ & $\left(0.915^{*}\right)$ & $\left(0.655^{*}\right)$ \\
\hline
\end{tabular}

* the ratio of identified value to true value and modal ground acceleration input, and then provides more precise modal characteristics; Tables 2 and 3 provide the obtained modal characteristics.

From the data shown in Tables 2 and 3 the transfer function vector considering up to the first three modes is calculated. Utilizing the obtained transfer function vector the input ground acceleration can be identified by means of Eq.(10). As already mentioned, the obtained transfer function only involves the information up to the frequency of the fundamental third modes in this case; hence the estimated ground acceleration involves the information from $0 \mathrm{~Hz}$ to 2.0



(a) Identified input acceleration from the 20th-story transfer function



(b) Identified input acceleration from the 14th-story transfer function

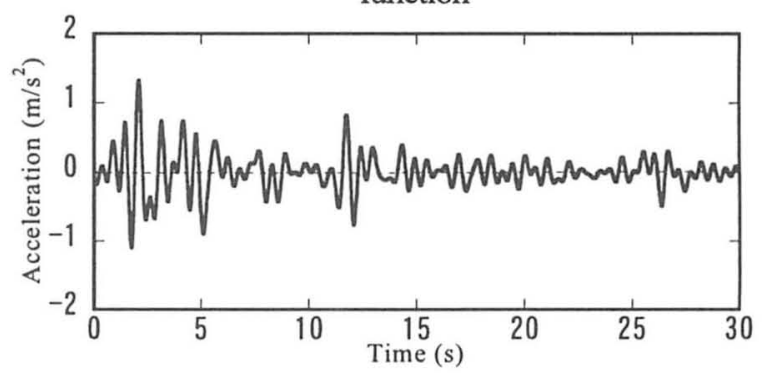

(c) Identified input acceleration from the 8 th-story transfer

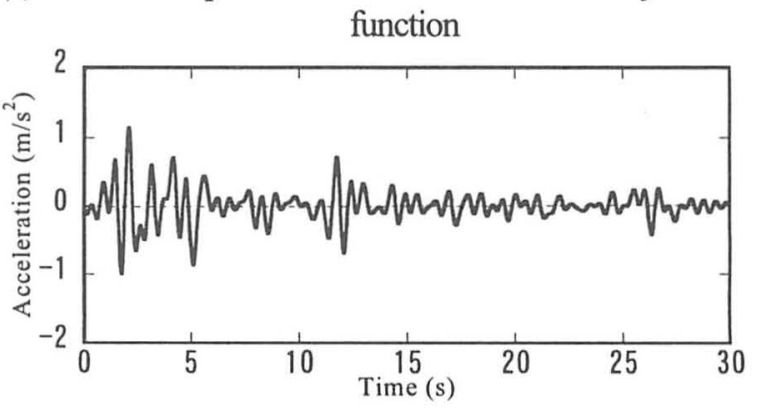

(d) Low-pass filtered input acceleration

Fig. 1 Comparison between identified and low-pass filtered input accelerations 
$\mathrm{Hz}$ in the frequency range i.e. it is the one which has been subjected to such a lowpass filter. Fig.1 compares the estimated ground acceleration time history with the real time history. An excellent agreement can be recognized. It can be also recognized that the transfer function for any story gives a quite accurate estimate of the ground acceleration.

\section{Experimental Verification}

Experiments are conducted using the scale model of a 6story building shown in Fig.2. This scale model is subjected to two kinds of seismic excitations: one is the NS component of the $1940 \mathrm{El}$ Centro earthquake and the other is the EW component of the 1968 Hachinohe earthquake. The peak ground accelerations of both earthquakes are $1.0 \mathrm{~m} / \mathrm{sec}^{2}$. The absolute acceleration data measured for the second, forth and sixth stories with the sampling time $\Delta t=0.005 \mathrm{sec}$ are utilized in performing the identification of this scale model building assuming that the ground accelerations are unknown. Since the output data are measured only for the second, forth and sixth of the scale model building for the experiments, the identified modal vectors is composed of the three components. The first, second and third components of each vector correspond to the second, forth and sixth stories.

All of the output data are decimated so as to have the time step interval in the discrete time calculation $\Delta t=0.025 \mathrm{sec}$ and the duration of $35 \mathrm{sec}$, and pass through the bandpass filters for the following passbands: $1.3 \mathrm{~Hz} \sim 1.8 \mathrm{~Hz}$ for the identification of the first mode; $4.6 \mathrm{~Hz} \sim 5.0 \mathrm{~Hz}$ for the identification of second mode; and $7.7 \mathrm{~Hz} \sim 8.2 \mathrm{~Hz}$ for the identification of the third mode. The waves subjected to the frequency ranges only for the vicinities of the natural frequencies are used in the POD analysis. The modal vectors thus obtained for two kinds of seismic excitations are given in Tables 4 and 5 for the El Centro and Hachinohe earthquakes, respectively. Based on those modal vector information provided in Tables 4 and 5, input earthquake accelerations are

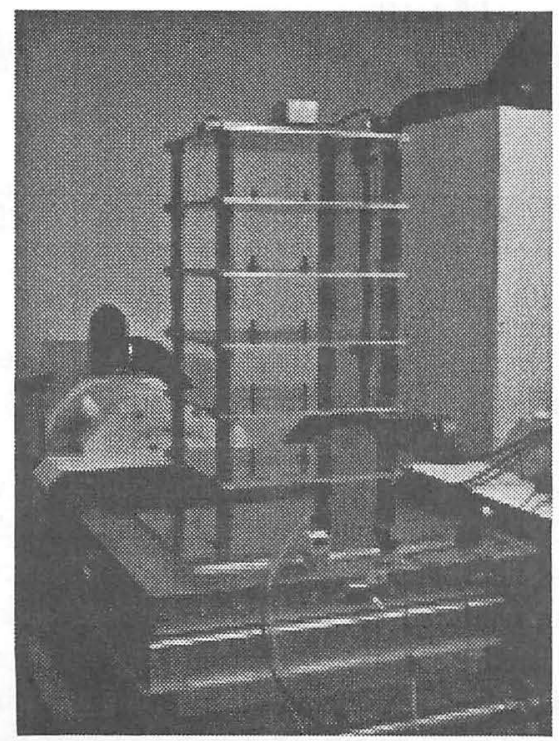

Fig. 2 Photograph of scale model building
Table 4 Modal vectors identified by POD for the $1940 \mathrm{El}$ Centro earthquake

\begin{tabular}{ccrr}
\hline Component & 1st mode & 2nd mode & 3rd mode \\
\hline 1st & 0.499 & 1.000 & 0.667 \\
2nd & 0.838 & 0.277 & -1.000 \\
3rd & 1.000 & -0.779 & 0.663 \\
\hline
\end{tabular}

Table 5 Modal vectors identified by POD for the 1968 Hachinohe earthquake

\begin{tabular}{ccrr}
\hline Component & 1st mode & 2nd mode & 3rd mode \\
\hline 1st & 0.497 & 1.000 & 0.633 \\
2nd & 0.836 & 0.271 & -1.000 \\
3rd & 1.000 & -0.776 & 0.646 \\
\hline
\end{tabular}

Table 6 Frequencies and dampings identified by ARX model for the 1940 El Centro earthquake

\begin{tabular}{ccc}
\hline Mode & $\begin{array}{c}\text { Natural frequency } \\
{[\mathrm{Hz}]}\end{array}$ & Damping ratio \\
\hline 1 & 1.549 & 0.0088 \\
2 & 4.809 & 0.0044 \\
3 & 7.888 & 0.0048 \\
\hline
\end{tabular}

Table 7 Modal vectors identified by ARX model for the 1940 El Centro earthquake

\begin{tabular}{ccrr}
\hline Component & 1st mode & 2nd mode & 3rd mode \\
\hline 1st & 0.596 & 0.347 & 0.057 \\
2nd & 1.004 & 0.103 & -0.107 \\
3rd & 1.195 & -0.276 & 0.081 \\
\hline
\end{tabular}

Table 8 Frequencies and dampings identified by ARX model for the 1968 Hachinohe earthquake

\begin{tabular}{ccc}
\hline Mode & $\begin{array}{c}\text { Natural frequency } \\
{[\mathrm{Hz}]}\end{array}$ & Damping ratio \\
\hline 1 & 1.567 & 0.0083 \\
2 & 4.814 & 0.0030 \\
3 & 7.733 & 0.0043 \\
\hline
\end{tabular}

Table 9 Modal vectors identified by ARX model for the 1968 Hachinohe earthquake

\begin{tabular}{ccrr}
\hline Component & 1st mode & 2nd mode & 3rd mode \\
\hline 1st & 0.598 & 0.335 & 0.067 \\
2nd & 1.002 & 0.096 & -0.097 \\
3rd & 1.205 & -0.265 & 0.060 \\
\hline
\end{tabular}

estimated by using the output acceleration responses subjected to the bandpass filters with the following passbands: $1.0 \mathrm{~Hz} \sim$ $2.1 \mathrm{~Hz}$ for the first mode; $4.3 \mathrm{~Hz} \sim 5.4 \mathrm{~Hz}$ for the second mode; and $7.3 \mathrm{~Hz} \sim 8.5 \mathrm{~Hz}$ for the third mode. The estimated input accelerations are the data subjected to the corresponding bandpass filters. The ARX model-based identification 




(a) Identified input acceleration from the 6th-story transfer function

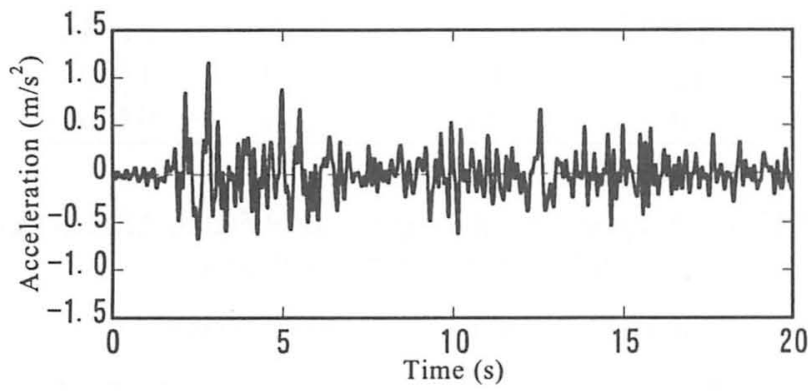

(b) Identified input acceleration from the 4th-story transfer function

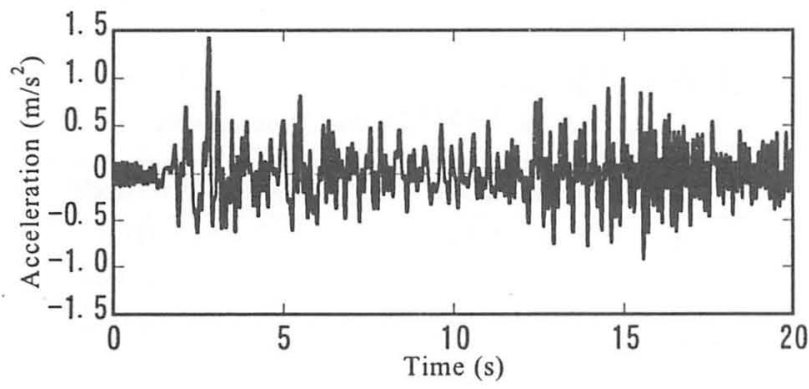

(c) Identified input acceleration from the 2nd-story transfer function

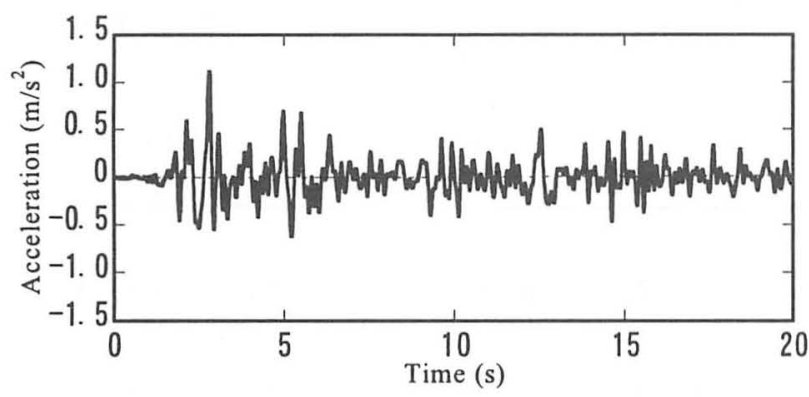

(d) Low-pass filtered input acceleration

Fig. 3 Comparison between identified and low-pass filtered input accelerations (El Centro 1940 NS)

technique is applied to thus obtained input and output data. The estimated modal characteristics are given in Tables 6 and 7 for the El Centro earthquake and in Tables 8 and 9 for the Hachinohe earthquake.

Utilizing the transfer functions calculated from these data in Tables 6-9, the input ground accelerations are estimated based on Eq.(10). In Figs.3 and 4, a variety of estimated input ground

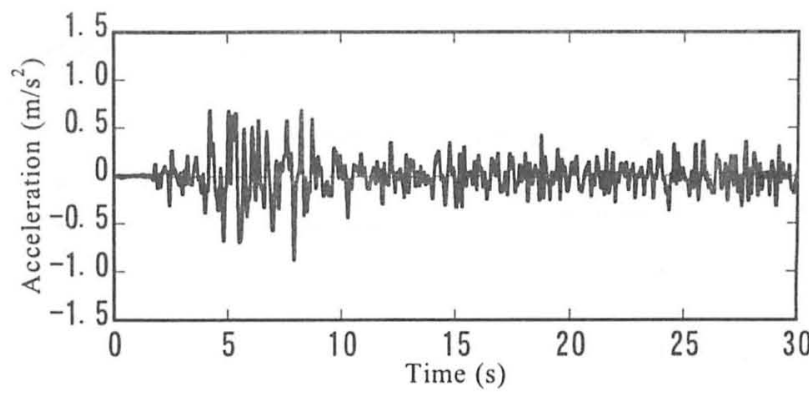

(a) Identified input acceleration from the 6th-story transfer function

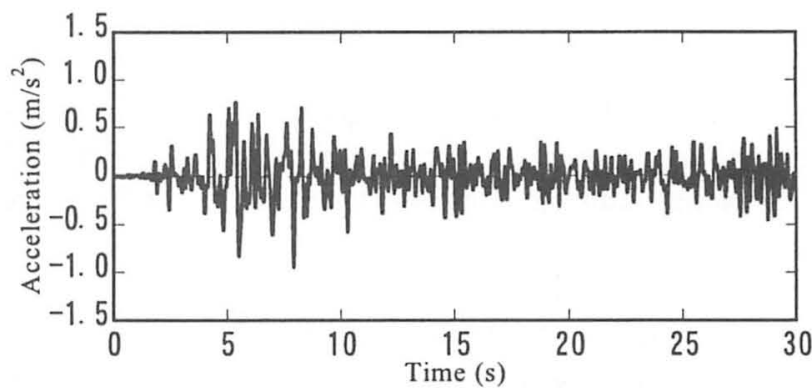

(b) Identified input acceleration from the 4th-story transfer function

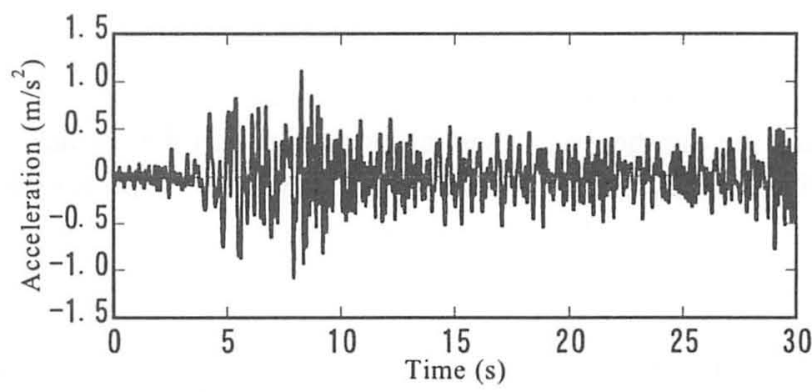

(c) Identified input acceleration from the 2nd-story transfer function

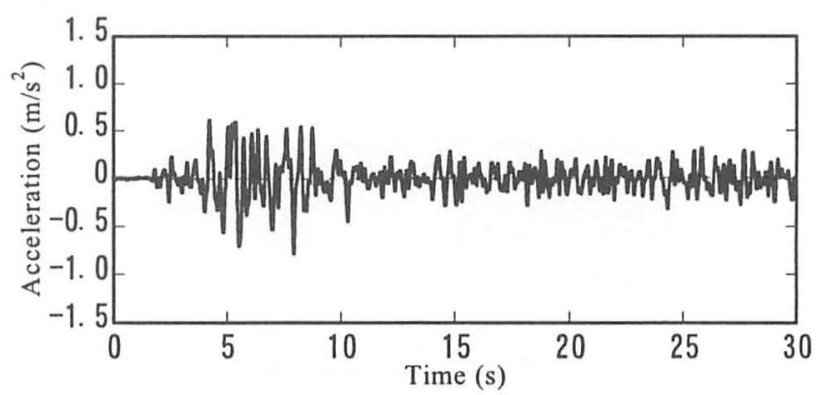

(d) Low-pass filtered input acceleration

Fig. 4 Comparison between identified and low-pass filtered input accelerations (Hachinohe $1968 \mathrm{EW}$ )

accelerations are shown, being compared with the theoretically obtained input ground accelerations subjected to the lowpass filter with the passband edge frequency $7.5 \mathrm{~Hz}$. These figures indicate that the transfer functions for the forth and sixth stories provide an accurate estimation of the lowpass-filtered input accelerations but the transfer functions for the second story do not. This might be from the fact the 


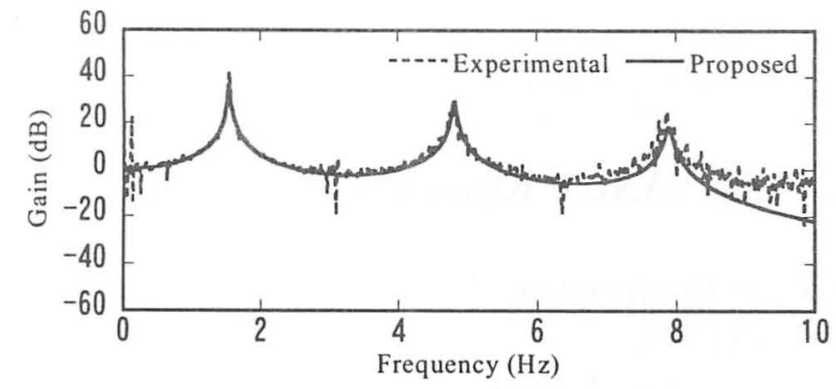

Fig. 5 Comparison of transfer functions (El Centro 1940 NS)

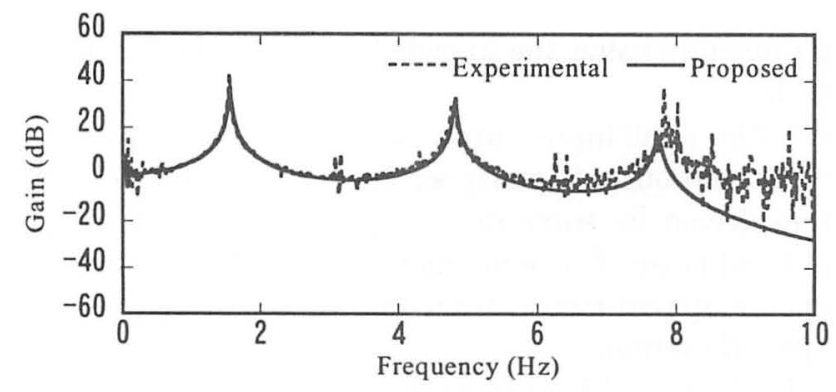

Fig. 6 Comparison of transfer functions (Hachinohe 1968 EW)

response of the second story is relatively small compared to the forth and sixth stories' responses and hence it is likely to be more affected by the measurement noise.

For the purpose of realizing how accurately the proposed methodology performs, the absolute values of those transfer functions calculated simply by dividing the Fourier transform of the absolute acceleration response of the top story by the Fourier transform of the input ground acceleration are compared with the corresponding values resulting from the proposed methodology in Figs.5 and 6. In these two figures, the dashed line indicating "Experimental" represents the ratio of the Fourier transforms of the experimentally obtained input and output, while the solid line indicating "Proposed" represents the proposed methodology-based identified transfer function. They are in good agreement in both of the figures.

\section{Conclusion}

This paper presents a structural identification methodology simply based upon the absolute acceleration response measurements for the selected stories of a structure. The proposed methodology can precisely provide the motion characteristics of a structure and can also estimate the input ground acceleration. The effectiveness of the proposed methodology has been demonstrated by conducting the computer simulations and scale model experiments.

\section{References}

[1] Y.Tamura, S.Suganuma, H.Kikuchi and K.Hibi (1999) Proper orthogonal decomposition of random wind pressure field, Journal of Fluids and Structures, No.13, $1069 / 1095$

[2] Y.Tamura (1995) An introduction of applying proper orthogonal decomposition to random filed, Journal of Wind Engineering, No.65, 33/41 (in Japanese)

[3] L.Ljung (1999) System Identification: Theory for User, Second Edition, Prentice Hall, Englewood, Cliffs, N.J

[4] T.Saito (1998) System identification of a high-rise building applying multi-input-multi-output ARX model of modal analysis, Journal of Structural and Construction Engineering(Transaction of AIJ), No.508, $47 / 54$ (in Japanese)

[5] A.Nishitani, Y.Nitta and S.Yamada (1999) Model reduction for active-controlled building structures, Proceedings of the Second World Conference on Structural Control, 2231/2240 